\title{
Alternative Magnetflussführung in Elektromotoren
}

\section{Reproduzierbar eingebrachte Eigenspannungen zur gezielten Magnetflussführung in nicht-kornorientiertem Elektroblech}

\author{
Benedikt Schauerte ${ }^{1}$ (D) Ines Gilch ${ }^{2} \cdot$ Tobias Neuwirth $^{3} \cdot$ Simon Sebold $^{3} \cdot$ Nora Leuning $^{1} \cdot$ Michael Schulz $^{3}$. \\ Wolfram Volk ${ }^{2}$ - Kay Hameyer ${ }^{1}$
}

Eingegangen: 22. Januar 2021 / Angenommen: 8. Mai 2021 / Online publiziert: 16. Juni 2021

(c) Der/die Autor(en) 2021

\section{Zusammenfassung}

Nicht-kornorientiertes Elektroblech ist eine Kernkomponente im Aufbau rotierender elektrischer Maschinen. Es dient der gezielten Führung und Verstärkung des magnetischen Flusses. Eine möglichst starke magnetische Kopplung zwischen Rotor- und Statorfluss ist hierbei entscheidend für die Effizienz und Leistungsdichte des elektrischen Antriebs.

$\mathrm{Zu}$ diesem Zweck werden in permanentmagneterregten Synchron-Maschinen und Synchron-Reluktanzmaschinen Flussbarrieren eingebracht, um Streuflüsse zu unterdrücken und eine gezielte Führung des magnetischen Flusses zu gewährleisten. Die Flussbarrieren werden typischerweise durch Aussparungen im Rotorquerschnitt realisiert. Die resultierenden filigranen Strukturen des Elektroblechs führen zu einer mechanischen Schwächung des Rotors und somit zu geringeren erreichbaren Drehzahlen und Leistungsdichten.

In dieser Arbeit wird ein alternativer Ansatz zur Führung des magnetischen Flusses auf Basis des Villari-Effekts vorgestellt. Lokal veränderte magnetische Werkstoffeigenschaften werden durch erhöhte Eigenspannungen in geprägten Werkstoffbereichen erzielt. Der Zusammenhang zwischen umformtechnisch eingebrachten Eigenspannungen und der reduzierten magnetischen Leitfähigkeit des Elektroblechs wird anhand homogen geprägter Proben untersucht. Höhere Umformkräfte, die mit gesteigerten mechanischen Spannungen einhergehen, führen zu einer stärkeren Reduktion der magnetischen Permeabilität. Die Funktionsweise dieser alternativen magnetischen Flusssperren wird anhand kreisförmiger Prägemuster gezeigt. Neben der Darstellung des Einflusses variierender Prägeparameter auf die magnetischen Eigenschaften des Elektroblechs wird vor allem die Reproduzierbarkeit der veränderten magnetischen Eigenschaften durch umformtechnisch hervorgerufene Eigenspannungen nachgewiesen.

\author{
Benedikt Schauerte \\ benedikt.schauerte@iem.rwth-aachen.de \\ Ines Gilch \\ ines.gilch@utg.de \\ Tobias Neuwirth \\ tobias.neuwirth@frm2.tum.de
}

1 Institut für Elektrische Maschinen (IEM), RWTH Aachen University, Schinkelstr. 4, 52062 Aachen, Deutschland

2 Lehrstuhl für Umformtechnik und Gießereiwesen (utg), Walther-Meißner-Str. 4, 85748 Garching, Deutschland

3 Heinz Maier-Leibnitz Zentrum (MLZ), Lichtenbergstr. 1, 85748 Garching, Deutschland 


\title{
Alternative magnetic flux guidance in rotating electrical machines
}

Reproducible inserted residual stress for targeted magnetic flux guidance in non-oriented electrical steel

\begin{abstract}
Non-oriented electrical steel is a core component in the construction of rotating electrical machines for the targeted guidance and amplification of the magnetic flux. A strong magnetic coupling between rotor and stator flux is crucial for the maximization of the efficiency and power density of the electric drives. For this purpose, flux barriers are inserted into permanent magnet excited synchronous machines and synchronous reluctance machines in order to suppress leakage flux and to ensure targeted guidance of the magnetic flux. The flux barriers are typically implemented through cutouts in the rotor cross-section. The resulting filigree structures of the electrical steel lead to a mechanical weakening of the rotor and thus to lower achievable speeds and power densities. In this work an alternative approach for guiding the magnetic flux based on the Villari effect is presented. Locally changed magnetic material properties are achieved through increased residual stresses in the embossed material areas. The relationship between residual mechanical stresses introduced by the forming process and the reduced magnetic permeability of the electrical steel sheet is investigated using homogeneously embossed samples. Higher forming forces associated with increased mechanical stresses lead to a greater reduction in magnetic permeability. The functionality of these alternative magnetic flux barriers is shown using circular embossed patterns. In addition to showing the influence of varying stamping parameters on the magnetic properties of the electrical steel sheet, the reproducibility of the changed magnetic properties due to residual stresses caused by the forming process is demonstrated.
\end{abstract}

\section{Einleitung}

Die Entwicklung effizienter Elektromotoren ist von großer Bedeutung für die Erhöhung des Anteils elektrifizierter Antriebskonzepte in der Energiewende. Hierbei werden aufgrund der angestrebten hohen Leistungsdichten meist permanentmagneterregte Synchronmaschinen (PMSM) und zunehmend auch Synchron-Reluktanzmaschinen (SRM) als Traktionsantriebe eingesetzt [1].

Bei diesen Maschinentopologien ist eine möglichst starke magnetische Kopplung zwischen Rotor und Stator entscheidend für das übertragbare Drehmoment des Elektromotors. Diese Kopplung wird durch Aussparungen im Elektroblech des Rotorquerschnitts erzwungen. Die Aussparungen wirken durch die wesentlich geringere magnetische Permeabilität der Luft im Vergleich zum Elektroblech als magnetische Flussbarriere und minimieren das Volumen des verbleibenden magnetisch leitfähigen Werkstoffs. Der Magnetfluss wird dadurch gezielt geführt und nachteilige Streuflüsse werden unterdrückt. Die im Betrieb auftretenden Zentrifugalkräfte und abzustützenden Drehmomente wirken konzentriert auf die verbleibenden schmalen Stege des Elektroblechs im Rotorquerschnitt. Die maximal erreichbaren Drehzahlen werden somit durch die Maßnahmen zur Steigerung der magnetischen Kopplung signifikant beschränkt. Die vorliegenden filigranen Rotorgeometrien weisen eine verringerte mechanische Stabilität auf, wodurch die maximal zulässige Zentrifugalkraft begrenzt und die erreichbare Drehzahl reduziert wird. Im Auslegungsprozess von PMSM [2] und SRM [3] muss demzufolge ein Kompromiss zwischen der Kopplung des magnetischen Kreises und der maximalen Drehzahl auf Basis der auftretenden Kräfte in der Rotorstruktur erfolgen.

Ziel dieser Arbeit ist die Untersuchung des Aufbaus von magnetischen Flussbarrieren durch gezielt in das weichmagnetische Material eingebrachte Eigenspannungen. Unter Ausnutzung des Villari-Effekts, der eine Abnahme der magnetischen Permeabilität unter dem Einfluss mechanischer Spannungen beschreibt, werden die magnetischen Materialeigenschaften durch Eigenspannungen lokal verändert. Dieses Phänomen erlaubt die gezielte Führung des magnetischen Flusses durch geprägte Flussbarrieren bei geringer mechanischer Schwächung der Rotorstruktur. Ziel dieser Arbeit ist die Quantifizierung des Effekts gezielt eingebrachter Eigenspannungen auf die magnetischen Eigenschaften eines nicht kornorientierten Elektroblechs. Hierfür werden Bleche mit homogenen Mustern aus Prägepunkten versehen, die mit unterschiedlichen Prozesskräften gefertigt werden. Ebenfalls wird der Effekt verschiedener Eigenspannungsverteilungen durch eine Variation des Prägemusters untersucht. Die magnetische Flussführung wird im Vergleich einer kreisförmigen Aussparung und eines kreisförmigen Prägemusters betrachtet. Hierbei wird neben einer Variation der Prägekraft vor allem Fokus auf eine reproduzierbare Einstellung der magnetischen Eigenschaften gelegt. 


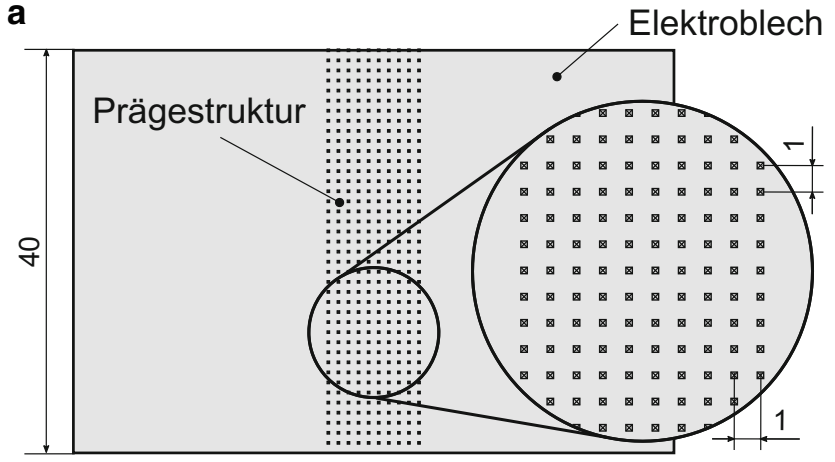

b

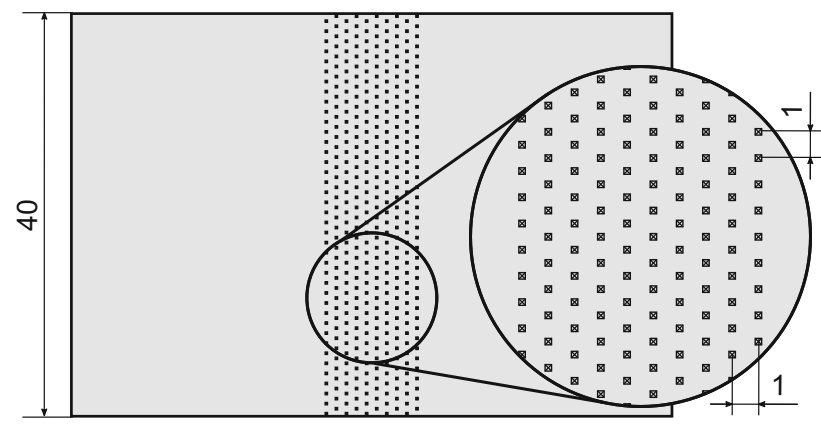

C

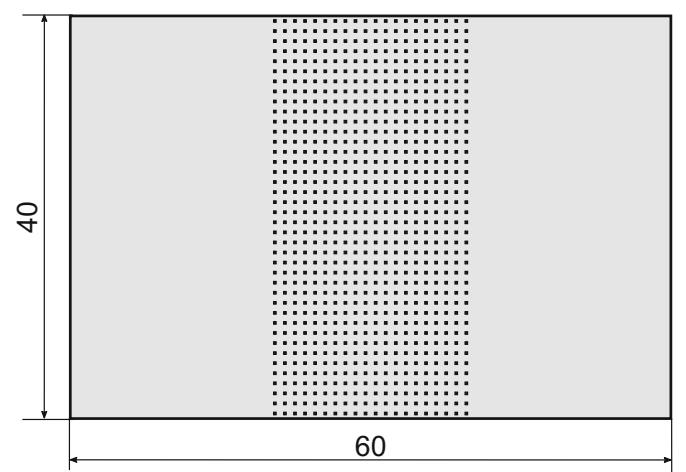

$10 \mathrm{~mm}$

Abb. 1 Schematische Darstellung der Probengeometrie mit homogener Prägung: a homogene Prägung; b homogene Prägung mit versetzten Prägelinien; $\mathbf{c}$ homogene Prägung mit doppelter Prägefläche

\section{Methoden}

\subsection{Prägeprozess - Lokale Veränderung des mechanischen Spannungszustands im Elektroblech}

Die untersuchte Methode zur alternativen Magnetflussführung in Elektroblechen basiert auf der lokalen Änderung des mechanischen Spannungszustands im Elektroblech. Durch das Prägen-eine Werkstoffdeformation in der Größenordnung von Mikrometern-werden lokal Eigenspannungen ins a

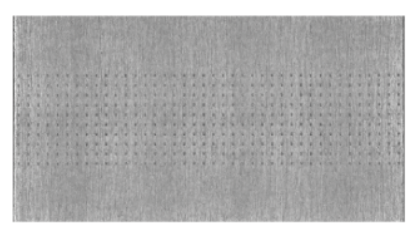

C

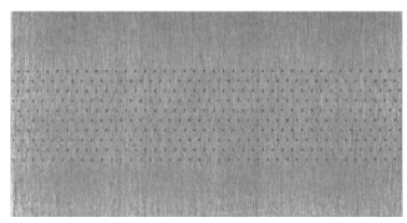

b

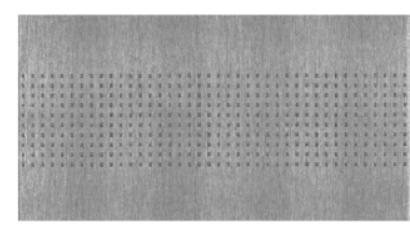

d

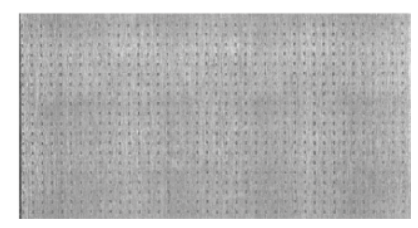

$10 \mathrm{~mm}$

Abb. 2 Nahaufnahmen der geprägten Bereiche der Probengeometrien mit homogener Prägung: a homogene Prägung mit 100 N Prägekraft; b homogene Prägung mit $200 \mathrm{~N}$ Prägekraft; c homogene Prägung mit versetzten Prägelinien mit 100 N Prägekraft; d homogene Prägung mit doppelter Prägefläche bei 100 N Prägekraft

Material eingebracht, wobei die Änderung der magnetischen Eigenschaften örtlich begrenzt ist.

Geprägt wird mit Prägestempeln, die aus einer, drei, vier oder zehn in Reihe angeordneter Pyramidenspitzen mit einem Spitzenwinkel von $136^{\circ}$ bestehen. Nach vorangegangenen Untersuchungen mit pyramiden-, zylinder-, kegelund kugelförmigen Prägegeometrien wurde die Pyramide als Stempelform gewählt, da die spitze Stempelgeometrie bereits bei geringen Prägekräften zu einer Plastifizierung des Werkstoffs führt. Vorerst wird auf kompliziertere Prägegeometrien - wie zum Beispiel längliche Eindrücke - verzichtet, da unsymmetrische Umformungen zu ungleichmäßigen Blechdurchbiegungen führen und durch ihre magnetische Anisotropie nur für Spezialfälle einsetzbar sind. Die Prägekraft beträgt je Pyramidenspitze 50N,

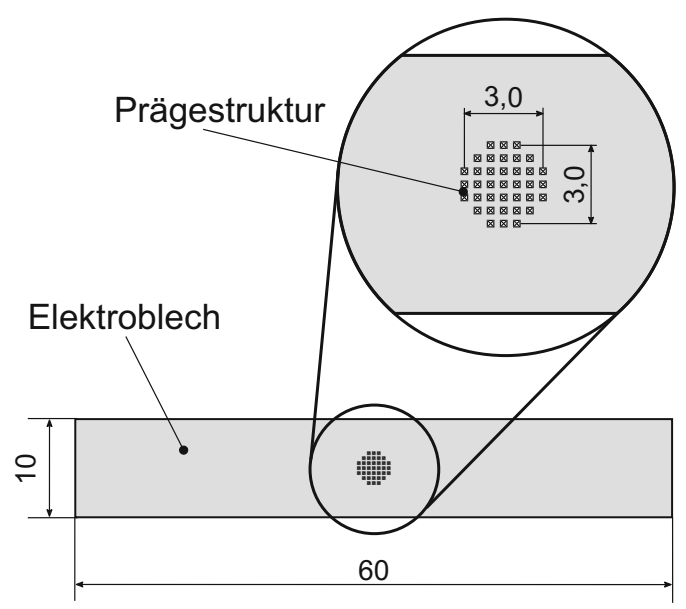

$10 \mathrm{~mm}$

Abb. 3 Schematische Darstellung der Probengeometrie mit Kreisprägung 
$100 \mathrm{~N}$ beziehungsweise 200 N. Anhand zweier Prägestrategien-homogene und kreisförmige Prägung-wird einserseits der Zusammenhang zwischen induzierten Eigenspannungen und der globalen Veränderung der magnetischen Eigenschaften analysiert, andererseits die Möglichkeit der magnetischen Flussführung in Elektroblechen durch geprägte Strukturen gezeigt. Die Untersuchungen werden an einem industriell eingesetzten, nicht-kornorientierten Elektroblech mit einer Blechdicke von 0,35 mm und 2,4 Gew.$\%$ Silizium durchgeführt. Die Magnetisierungsrichtung der Bleche entspricht bei allen Versuchen der Walzrichtung. Die Streckgrenze des weichmagnetischen Materials beträgt $363 \mathrm{MPa}$ und die Zugfestigkeit 464 MPa [4].

Um den Effekt des Prägeprozesses auf die Magnetisierbarkeit bewerten zu können, wird eine homogene Prägestruktur auf einer Probe der Größe $40 \mathrm{~mm} \times 60 \mathrm{~mm}$ geprägt. Die Probengeometrie wird mittels Scherschneiden zugeschnitten, da das Trennverfahren aufgrund der großen Probenbreite einen vergleichsweise geringen Einfluss auf das magnetische Verhalten der gesamten Probe hat [5]. Als Referenz wird eine ebenfalls schergeschnittene Probe gewählt. Der Einfluss der Prägekraft; 100 N und 200 N je Prägepunkt; wird an einer homogenen Prägestruktur aus 10 Prägelinien mit je 40 Prägepunkten untersucht, siehe Abb. 1a.

Die Prägepunkte haben einen Abstand von 1,0 mm. Die sequentiell hinzugefügten geprägten Linien sind senkrecht zum angelegten magnetischen Feld orientiert. Die Probenbezeichnung setzt sich aus der Anzahl der geprägten Linien $n_{\text {Linie }}$, der Anzahl der Prägepunkte je Linie $n_{\text {Prägepunkte }}$ und der Prägekraft je Prägepunkt $F_{\text {Prägepunkt }}$ zusammen: $n_{\text {Linie }} \times n_{\text {Prägepunkte }}-F_{\text {Prägepunkt }}$.

Inwieweit die Anordnung der Prägepunkte einen Einfluss auf die magnetischen Eigenschaften besitzt, wird anhand eines homogenen Prägemusters analysiert, wobei jede zweite Linie um $0,5 \mathrm{~mm}$ versetzt geprägt wird, wie in Abb. $1 \mathrm{~b}$ dargestellt. Hierbei beträgt die Prägekraft je Prägepunkt $100 \mathrm{~N}$, weswegen die Proben mit 10x40-100N-2 bezeichnet werden.

Ebenso soll gezeigt werden, dass die Anzahl der geprägten Punkte bei ansonsten gleichbleibenden Randbedingungen - Prägekraft $100 \mathrm{~N}$ je Prägepunkt und 1,0 mm Abstand der Prägepunte - zu einer weiteren Erhöhung des magnetischen Widerstands führt. Wie in Abb. 1c skizziert, wird hierfür eine doppelt so große Fläche mit Prägepunkten versehen. Nahaufnahmen der unterschiedlich geprägten Bereiche bei den verschiedenen Prägekräften und Prägegeometrien ist in Abb. 2 dargestellt.

Die Analyse der magnetischen Flussführung, findet anhand einer annähernd kreisförmigen Prägung statt, die nachfolgend als Kreisprägung bezeichnet wird. Hierbei sind 37 Prägepunkte - wie in Abb. 3 dargestellt - mit einem Abstand von $0,5 \mathrm{~mm}$ angeordnet.
Anhand dieses Prägemusters, geprägt mit einer Prägekraft von $100 \mathrm{~N}$ je Prägepunkt, wird die Reproduzierbarkeit der eingestellten magnetischen Eigenschaften der Flussbarriere gezeigt. Hierfür werden fünf mit gleichen Prozessparametern hergestellte Proben miteinander verglichen. Außerdem wird erneut eine Variation der Prägekraft; $50 \mathrm{~N}, 100 \mathrm{~N}$ und 200 N je Prägepunkt; durchgeführt. Die Probenbezeichnung setzt sich bei den Kreisprägungen (Cyl.) mit der Prägekraft $F_{\text {Prägepunkt }}$ wie folgt zusammen: Cyl. $F_{\text {Prägepunkt }}$. Die identisch hergestellten Proben werden durch die angehängten Buchstaben A, B, C, D und E unterschieden.

Die für die Kreisprägungen verwendete Probengeometrie von $10 \mathrm{~mm} \times 60 \mathrm{~mm}$ ist mittels Drahterosion geschnitten, um einen möglichst geringen Effekt des Trennprozesses auf die magnetischen Eigenschaften der deutlich schmaleren Probe zu erzielen [5]. Die Probenvorbereitung entspricht den vorangegangenen Studien, jedoch unterscheidet sich der Prägeprozess durch die deutlich geringere Werkstoffdeformation verglichen zu den zuvor untersuchten makroskopischen Prägungen [6, 7].

Die Prägetiefe wird im Anschluss an den Prägeprozess mit einem Laserkonfokalmikroskop bestimmt. Bei den Kreisprägungen mit einer Prägekraft von $100 \mathrm{~N}$ ergibt sich eine mittlere Prägetiefe von $41,6 \mu \mathrm{m}$, wobei die Standardabweichung zwischen den einzelnen Proben $0,25 \mu \mathrm{m}$ ist. Die Prägetiefe bei $50 \mathrm{~N}$ je Prägepunkt beträgt $28,5 \mu \mathrm{m}$ und $62,9 \mu \mathrm{m}$ bei 200 N. Für die homogenen Prägungen sind die gemittelten Prägetiefen $40,7 \mu \mathrm{m}$ für $100 \mathrm{~N}$ Prägekraft und $62,4 \mu \mathrm{m}$ für $200 \mathrm{~N}$.

\subsection{Charakterisierung weichmagnetischer Materialien}

Die in dieser Arbeit vorgestellten magnetischen Messergebnisse wurden mithilfe eines $60 \times 60 \mathrm{~mm}$ Single-Sheet Tester (SST) durchgeführt [8]. Die Probe wird hierbei in den Zwischenraum des doppelt ausgeführten magnetischen Rückschlussjochs eingelegt, wodurch der magnetische Kreis geschlossen wird. Primär- und Sekundärwicklung umschlieBen die Probe und ermöglichen das Anlegen einer magnetischen Feldstärke $H$ und die Messung der resultierenden magnetischen Flussdichte $B$. Der zeitliche Verlauf der magnetischen Feldstärke $H$ wird durch die Messsoftware so geregelt, dass sich sinusförmige Flussdichten bei vorgegebener magnetischer Aussteuerung $B_{\mathrm{m}}$ und Magnetisierungsfrequenz $f$ einstellen. Die Messungen wurden in $0,1 \mathrm{~T}$-Schritten zwischen 0,1 und $1,8 \mathrm{~T}$ bei Magnetisierungsfrequenzen von 50,100, 200 und $400 \mathrm{~Hz}$ durchgeführt. 
Die im Folgenden dargestellten relativen magnetischen Permeabilitäten $\mu_{\mathrm{r}}$ errechnen sich jeweils aus den Umkehrpunkten der 18 gemessenen Hystereschleifen:

$\mu_{\mathrm{r}}=\frac{B_{\mathrm{m}}}{\mu_{0} \cdot H_{\mathrm{m}}}$

Um einen Versatz zwischen Proben und Rückschlussjoch und eine daraus resultierende Vergrößerung des magnetischen Widerstands zu vermeiden, wurde bei der Charakterisierung der $10 \times 60 \mathrm{~mm}$ Proben ein Vorrichtung zur mittigen Ausrichtung der Proben in das Doppeljoch des SSTs eingelegt. Hierdurch wird eine Wiederholbarkeit der Probenpositionierung in der Mitte des Doppeljochs mit paralleler Ausrichtung der Kanten zur Magnetisierungsrichtung sichergestellt. Eine Beeinflussung der Messergebnisse durch einen schwankenden magnetischen Übergangswiderstand zwischen Probe und Doppeljoch kann somit ausgeschlossen werden. Magnetische Messungen nach dem SST-Messprinzip erlauben grundsätzlich keine ortsaufgelöste Messung der magnetischen Eigenschaften. Das gesamte Materialvolumen der Probe geht in die erzielten Ergebnisse ein, weshalb die SST-Messungen als global, über das gesamte Probenvolumen gemittelt, verstanden werden können.

Zusätzlich zu den globalen Ergebnissen der SST-Messungen können mit Hilfe der Neutronengitterinterferometrie (nGI) Informationen über die lokalen magnetischen Eigenschaften gewonnen werden [9]. nGI ist eine fortschrittliche Technik im Bereich der Neutronenbildgebung. Hierbei nutzt man, dass die nGI die Streuung der Neutronen an mikrometergroßen magnetischen Strukturen ortsaufgelöst detektiert. Dadurch können lokale Änderungen der magnetischen Eigenschaften sichtbar gemacht werden. Das resultierende

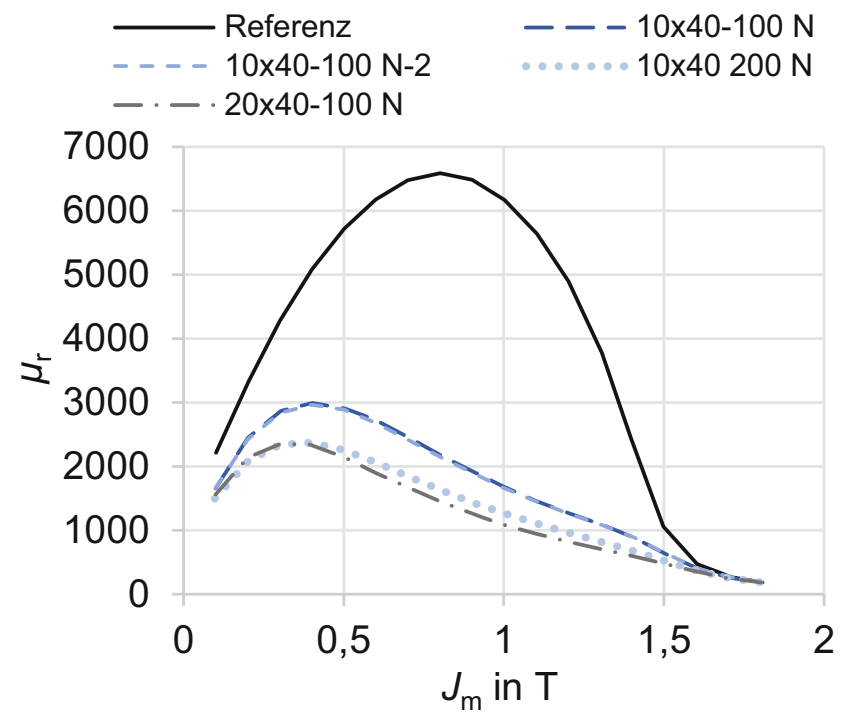

Abb. 4 Einfluss verschiedener homogen verteilter Prägekräfte und Geometrien auf die relative magnetische Permeabilität $\mu_{\mathrm{r}}$ in Abhängigkeit der Umkehrpolarisation $\boldsymbol{J}_{\mathrm{m}}$
Tab. 1 Relative Permeabilität der Referenzprobe $\mu_{\mathrm{r}, \text { ref }}$ und relative Permeabilität der geprägten Proben im Verhältnis zur Referenzprobe homogen geprägter Proben bei $0,5,1,0$ und 1,5 T

\begin{tabular}{lrrrr}
\hline Probe & \multicolumn{3}{c}{$J_{\mathrm{m}}$} \\
\cline { 2 - 4 } Referenz & $0,5 \mathrm{~T}$ & $1,0 \mathrm{~T}$ & $1,5 \mathrm{~T}$ & \\
\hline $10 \times 40-100 \mathrm{~N}$ & 5726 & 6173 & 1058 & $\mu_{\mathrm{r}, \text { ref }}$ \\
$10 \times 40-100 \mathrm{~N}-2$ & 0,507 & 0,272 & 0,583 & $\frac{\mu_{\mathrm{r}}}{\mu_{\mathrm{r}, \mathrm{ref}}}$ \\
$10 \times 40-200 \mathrm{~N}$ & 0,504 & 0,270 & 0,597 & \\
$20 \times 40-100 \mathrm{~N}$ & 0,394 & 0,204 & 0,596 & \\
\hline
\end{tabular}

Streubild wird dark field image (DFI) genannt. Im Fall der untersuchten Elektrobleche wird die magnetische Domänenwanddichte analysiert, welche direkt mit der magnetischen Flussdichte und dem Eigenspannungszustand korreliert [7]. In der von uns verwendeten Konvention senkt eine Erhöhung der Domänenwanddichte und die damit einhergehende Erhöhung der Streuung das Signal ab. Im Gegensatz dazu verringert eine geringere Materialdicke die Streuung und erhöht daher das Signal. In Neuwirth et al. [10] sind das verwendete Gitterinterferometer und die Messparameter genauer erläutert. Die nGI-Messungen wurden am Instrument ANTARES am FRM II in München durchgeführt [11, 12].

Die Kombination aus globalen SST-Messungen und lokalen nGI-Messungen erlaubt eine genaue Charakterisierung der vermessenen Proben. Entscheidende Kriterien für die quantitative Analyse und Bewertung der unterschiedlich geprägten Proben sind die Absenkung der Magnetisierbarkeit sowie die resultierenden Eisenverlustdichten $P_{\mathrm{Fe}}$. Die in dieser Arbeit abgebildeten SST-Messergebnisse beschränken sich auf die bei $50 \mathrm{~Hz}$ durchgeführten Messun-

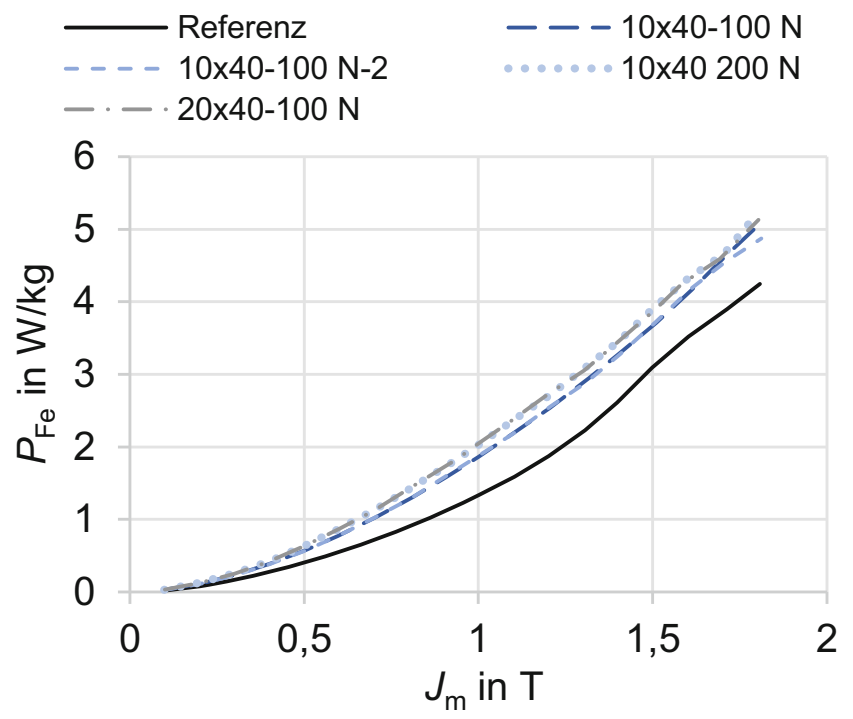

Abb. 5 Einfluss verschiedener homogen verteilter Prägekräfte und Geometrien auf die Eisenverlustdichte $P_{\mathrm{Fe}}$ in Abhängigkeit der Umkehrpolarisation $J_{\mathrm{m}}$ bei $50 \mathrm{~Hz}$ 


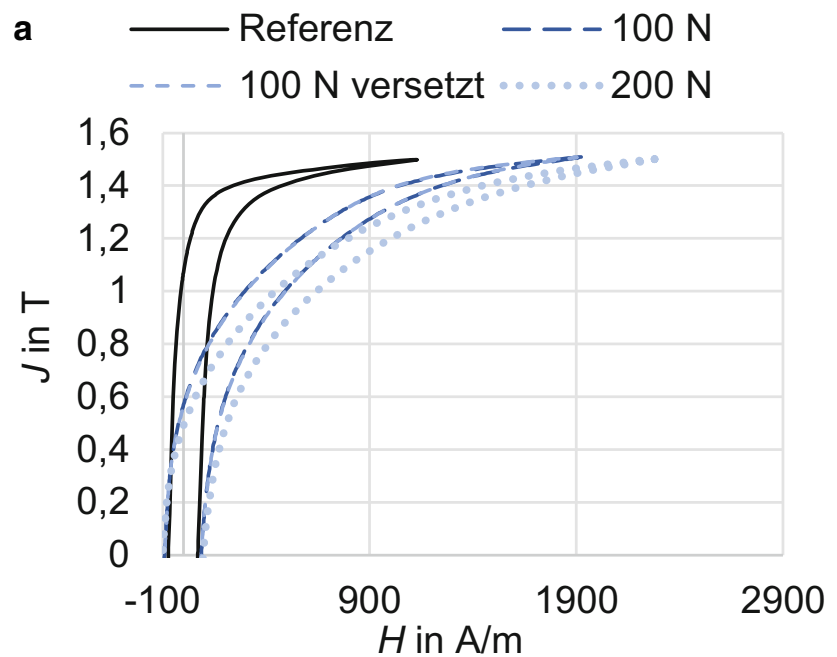

b
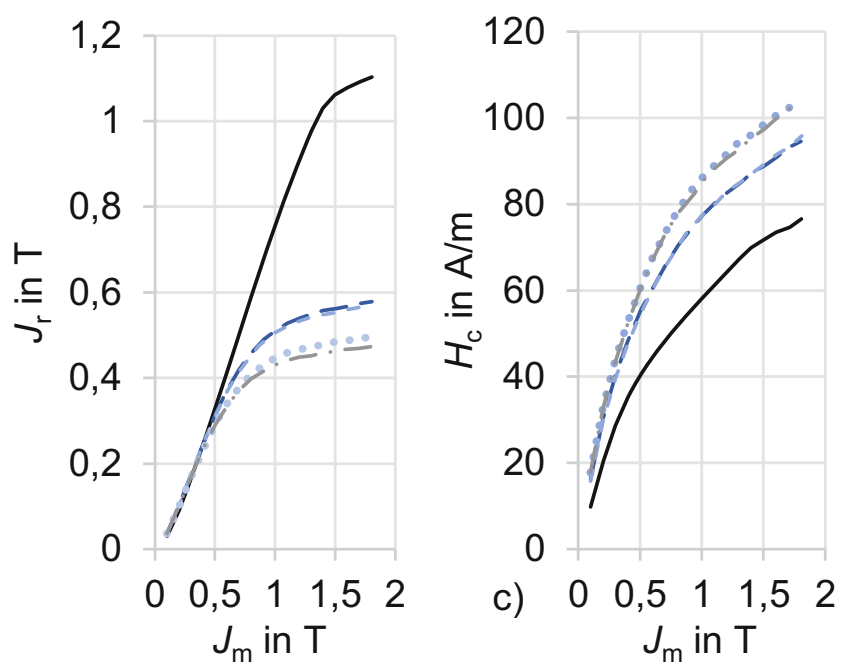

Abb. 6 Hystereseschleifen homogen geprägter Proben in a Remanenzpolarisation $J_{\mathrm{r}}$ in b und Koerzitivfeldstärke $H_{\mathrm{c}}$ c bei $50 \mathrm{~Hz}$ und Aussteuerung bis $1,5 \mathrm{~T}$

gen, da bei dieser Frequenz sowohl die Magnetisierungsverluste als auch die Magnetisierbarkeit am wenigsten durch spannungsunabhängige Wirbelströme beeinflusst werden, welche bei höheren Frequenzen vermehrt auftreten.

\section{Ergebnisse}

\subsection{Weichmagnetische Eigenschaften der homogenen Prägung}

Die homogen geprägten Proben erlauben die globale Charakterisierung veränderter magnetischer Eigenschaften durch umformtechnisch eingebrachte Eigenspannungen. Hierbei liegt kein Einfluss von umliegenden, nicht-geprägten Bereichen vor, auf die der magnetische Fluss ausweichen kann. In geeigneter Skalierung können somit repräsentative Volumenelemente definiert werden, deren Magnetisierbarkeit und Verlustverhalten durch die magnetischen Messungen der homogen geprägten Proben bestimmt werden können. Das Potential umformtechnisch eingebrachter Eigenspannungen zum Aufbau magnetischer Flussbarrieren kann somit ohne Beeinflussung ungeprägter, umliegender Bereiche beurteilt werden.

In Abb. 4 ist die relative magnetische Permeabilität $\mu_{\mathrm{r}}$ über der Umkehrpolarisation $J_{\mathrm{m}}$ aufgetragen. Die unbearbeitete Referenzprobe weist eine maximale Permeabilität von 6600 bei 0,8 Tesla auf. Die relative Permeabilität der sequentiell geprägten Proben mit 10 Prägelinien und $100 \mathrm{~N}$ Prägekraft sind in ihrer maximalen Magnetisierbarkeit bereits um mehr als $50 \%$ auf 3000 bei $0,4 \mathrm{~T}$ reduziert. Auffallend ist, dass zwischen der regelmäßig geprägten Probe und der versetzt geprägten Probe über den gesamten Messbereich keine klare Abweichung der Permeabilität erkennbar ist.

Die Probe mit 10 Linien und einer Prägekraft von 200 N weist eine maximale relative Permeabilität von 2375 bei 0,4 T auf. Auffallend ist hierbei, dass die Probe mit 20 Prägungslinien und $100 \mathrm{~N}$ Prägekraft mit einer Permeabilität von 2375 bei $0,4 \mathrm{~T}$ einen fast identischen Wert für die maximale Magnetisierbarkeit aufweist. Im weiteren Verlauf bis zum Erreichen der maximalen Umkehrpolarisation ist die Permeabilität der Probe mit 20 Prägelinien sogar durschnittlich um 150 niedriger als die mit $200 \mathrm{~N}$ geprägte Probe. Bei der Betrachtung des gesamten Verlaufs fällt auf, dass sich der Punkt der maximalen Permeabilität immer weiter in den Bereich niedrigerer Umkehrpolarisationen $J_{\mathrm{m}}$ bewegt. Dieser Effekt ist bereits bei vorangegangenen Untersuchungen zylindrischer Makroprägungen aufgetreten [7].

In Tab. 1 sind Zahlenwerte der relativen Permeabilität bei 0,5, 1,0 und 1,5 T angegeben. Bei Vergleich der Permeabilitäten bei identischen Umkehrpolarisationen treten bei 1,0 T Verringerungen der Magnetisierbarkeit des Materials auf weniger als $20 \%$ des Referenzwertes auf.

Die resultierenden Eisenverlustdichten bei $50 \mathrm{~Hz}$ sind in Abb. 5 dargestellt. Hier zeigen sich mit zunehmender Prägekraft und Prägepunktanzahl ansteigende Eisenverluste, die in guter Übereinstimmung zu dem auf Basis des Villari-Effektes zu erwartendem Verhalten stehen. Auffällig ist, dass zwischen den auftretenden Eisenverlusten der mit 10 Linien bei jeweils $200 \mathrm{~N}$ geprägten Probe und der Variante mit $100 \mathrm{~N}$ aber doppelter Prägepunktanzahl fast kein Unterschied auszumachen ist. Dem gegenüber steht eine über einen weiten Bereich reduzierte Permeabilität der 20x40-100N-Probe. Eine Verdopplung der geprägten Fläche scheint somit stärker auf die Magnetisierbarkeit zu wirken als eine Verdopplung der Prägekraft bei gleichbleibender Prägefläche. Der Zusammenhang, insbesondere unter Berücksichtigung der resultierenden Eisenverlustdichte, er- 


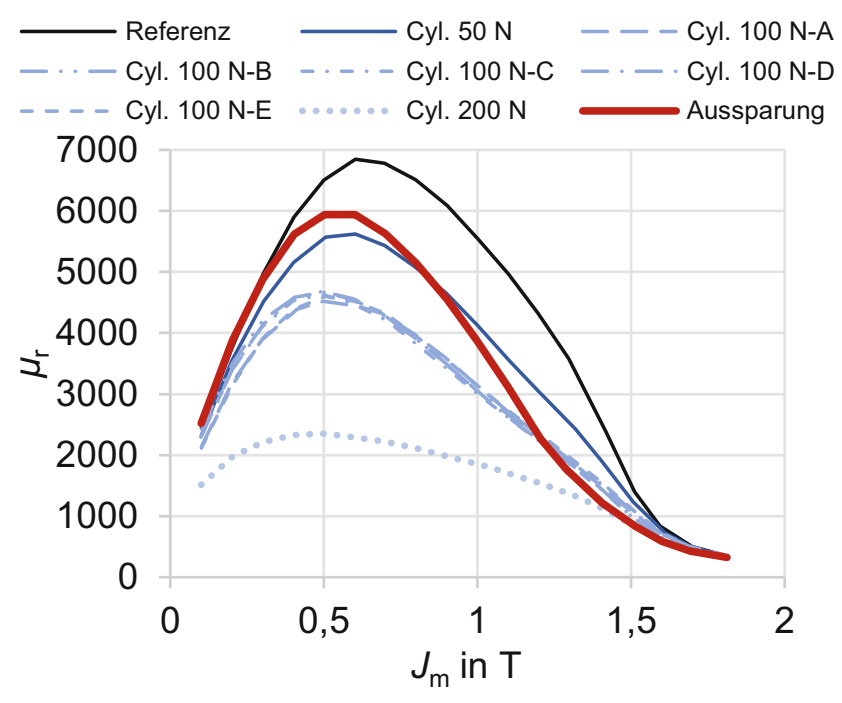

Abb. 7 Relative Permeabilität $\mu_{\mathrm{r}}$ sequentiell ausgeführter kreisförmiger Prägegeometrien gegen unbearbeitete Referenzprobe und ausgespartem Kreis bei $50 \mathrm{~Hz}$

fordert jedoch tiefergehende Untersuchungen bei weiteren Kombinationen der Prägeparameter. Im Gegensatz zum abweichenden Verhalten zwischen stärkerer Prägekraft und größerer Prägefläche, scheint die Anordnung der einzelnen Prägepunkte einen geringen Einfluss auf die Eisenverlustdichte auszuüben.

Zur genaueren Untersuchung des Einflusses des Prägemusters sind in Abb. 6 die Hysteresekurven der homogen geprägten Proben dargestellt. Sowohl bei den auf- als auch bei den absteigenden Kurven ist kein eindeutiger Unterschied zwischen den beiden Proben - nicht versetzt geprägt und versetzt geprägt - erkennbar. Zur weiteren Verdeutlichung sind die charakteristischen Werte der Remanenzpolarisation $J_{\mathrm{r}}$ und die Koerzitivfeldstärke $H_{\mathrm{c}}$ in Abb. 6a und b dargestellt. Auch hier tritt keine eindeutige Differenz zwischen einheitlichem und versetzem Prägemuster auf. Der Schluss liegt nahe, dass die Anordnung der Prägepunkte zumindest bei vorliegendem Abstand, Verteilung und Prägekraft, einen untergeordneten Einfluss auf die Eigenschaften der magnetischen Flussbarriere ausübt. Eine breitere

Tab. 2 Relative Permeabilität $\mu_{\mathrm{r}}$ homogen geprägter Proben bei 0,5, 1,0 und $1,5 \mathrm{~T}$

\begin{tabular}{|c|c|c|c|c|}
\hline \multirow[t]{2}{*}{ Probe } & \multicolumn{3}{|l|}{$J_{\mathrm{m}}$} & \\
\hline & $\overline{0,5 \mathrm{~T}}$ & $1,0 \mathrm{~T}$ & $1,5 \mathrm{~T}$ & \\
\hline Cyl. 100N-A & 4522 & 3136 & 1075 & $\mu_{\mathrm{r}}$ \\
\hline Cyl. 100N-B & 4681 & 3047 & 1101 & \\
\hline Cyl. 100 N-C & 4625 & 3004 & 991 & \\
\hline Cyl. 100N-D & 4667 & 3055 & 1004 & \\
\hline Cyl. 100N-E & 4599 & 3138 & 1129 & \\
\hline Std.-Abw. & 62,89 & 59,09 & 60,20 & \\
\hline
\end{tabular}
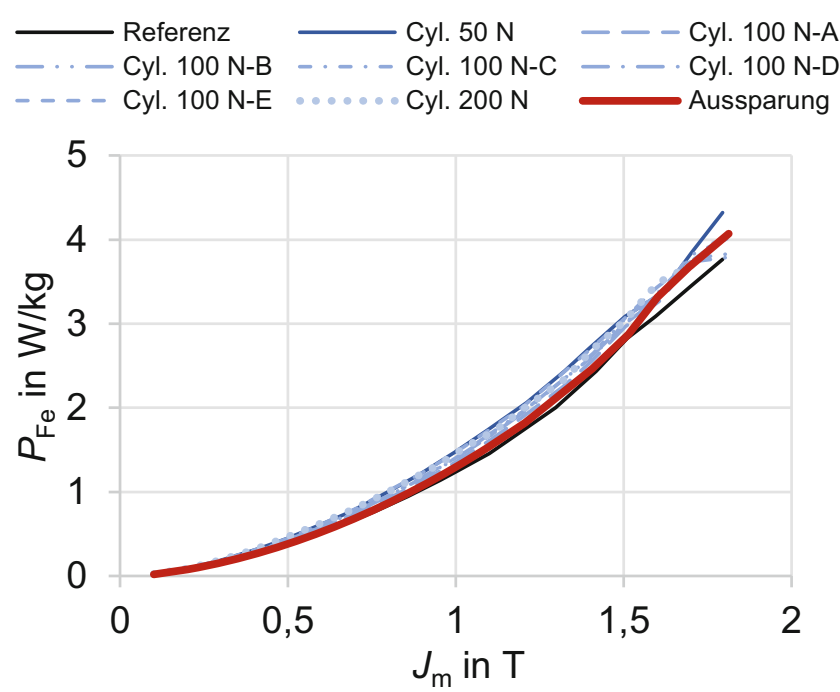

Abb. 8 Eisenverlustdichte $P_{\mathrm{Fe}}$ sequentiell ausgeführter kreisförmiger Prägemuster gegen unbearbeitete Referenzprobe und ausgesparten Kreis bei $50 \mathrm{~Hz}$

Variation genannter Prägeparameter ist jedoch notwendig um die Aussage allgemeingültig verifizieren zu können.

\subsection{Weichmagnetische Eigenschaften der Kreisprägung}

\subsubsection{Reproduzierbarkeit magnetischer Flussbarrieren durch umformtechnisch eingebrachte Eigenspannungen}

Entscheidendes Kriterium für die Nutzung umformtechnisch induzierter Eigenspannungen zum Aufbau elektromagnetischer Flussbarrieren ist die Stabilität und Reproduzierbarkeit des Herstellungsprozesses. Um neben den magnetischen Eigenschaften der Flussbarriere auch deren reproduzierbare Einstellbarkeit nachzuweisen, wurden unter identischen Prozessparametern hergestellte Proben mit Kreisprägungen nach Abb. 3 vermessen. Neben der unbehandelten Referenzprobe wurde eine Probe mit einer kreisförmigen Aussparung mit Durchmesser 3,0 mm gefertigt [6], um eine Vergleichbarkeit mit der herkömmlichen Aufbauweise magnetischer Flussbarrieren herzustellen. Die sequentiell geprägten Proben wurden jeweils einmal mit 50N und 200N geprägt. Zur Untersuchung der Reproduzierbarkeit des Prozesses wurden insgesamt fünf Proben unter gleichen Prozessparametern mit $100 \mathrm{~N}$ pro Prägepunkt hergestellt. Diese Proben werden im Folgenden durch die Laufbuchstaben A-E gekennzeichnet.

In Abb. 7 ist die Permeabilität der Proben aufgetragen. Wie zu erwarten, zeigt die unbearbeitete Referenzprobe für alle Umkehrpolarisationen $J_{\mathrm{m}}$ die höchste Permeabilität. Bemerkenswert ist, dass bereits die mit $50 \mathrm{~N}$ geprägte 
Probe für den Bereich niedrigerer Polarisationen einen höheren magnetischen Widerstand aufweist als die Probe mit der Aussparung. Dieser Effekt tritt in stärkerer Form bei sämtlichen mit $100 \mathrm{~N}$ geprägten Proben auf. Der magnetische Widerstand ist deutlich erhöht. Erst ab 1,3 T weist die ausgesparte Probe einen höheren magnetischen Widerstand auf. Die Messung der mit 200 N geprägten Probe bestätigt diesen Trend. Die magnetische Leitfähigkeit der Anordnung ist im Vergleich zur Aussparung um mehr als 50\% reduziert.

Hinsichtlich der Reproduzierbarkeit der Auswirkungen prägungsinduzierter Eigenspannungen auf die magnetischen Eigenschaften der Cyl. 100N-Proben ist eine deutliche Übereinstimmung in Verlauf und Amplitude der relativen Permeabilität $\mu_{\mathrm{r}}$ zu erkennen. Im anwendungstechnisch relevanten Bereich um 0,5 T verlaufen die Kurven nahezu identisch. Selbst im Bereich niedrigerer Magnetisierungen unter 0,5 T liegt die maximale relative Abweichung einer einzelnen Permeabilitätskurve vom Mittelwert unter $8 \%$. Die relativen Permeabilitäten und die jeweilige Standardabweichung sind für charakteristische Punkte in Tab. 2 angegeben. Die Standardabweichung gibt die durchschnittliche Abweichung vom Mittelwert der relativen Permeabilitität an und ist für alle drei abgebildeten Fälle im Verhältnis zu den absoluten Werten vernachlässigbar. Die Differenz zu den mit 50 und $200 \mathrm{~N}$ geprägten Proben liegt ebenfalls deutlich über den bei den identisch hergestellten Proben beobachteten Schwankungen in der Messung.

Bei Betrachtung der resultierenden Eisenverluste $P_{\mathrm{Fe}}$ in Abb. 8 ergibt sich ein deutlich einheitlicheres Bild als bei der Magnetisierung. Wie zu erwarten hat die unbearbeitete Referenzprobe über den gesamten Bereich die niedrigsten Verluste. Alle geprägten Proben weisen höhere Verluste als die unbearbeitete Referenzprobe und die Probe mit kreisförmiger Aussparung auf. Ein grundsätzlich erwartba-

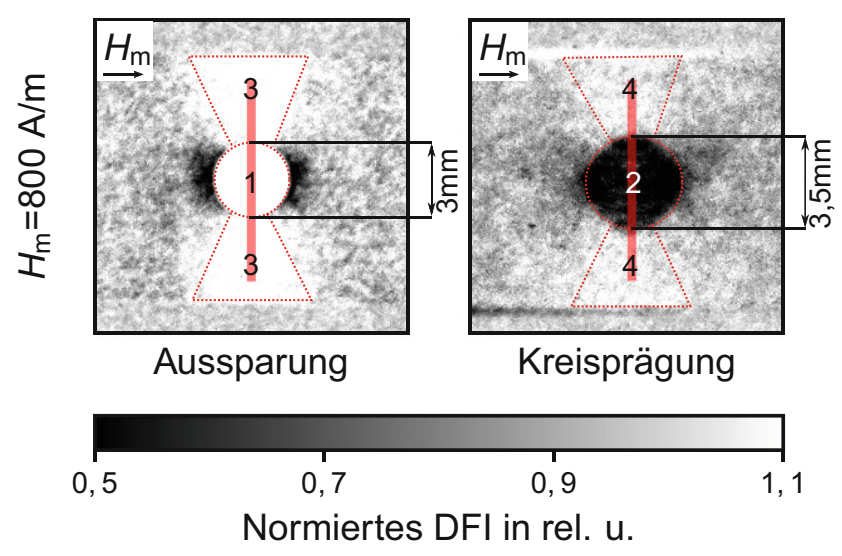

Abb. 9 Vergleich der Verdrängung des magnetischen Flusses durch Aussparung und Kreisprägung bei einem angelegtem magnetischen Feld von $H_{\mathrm{m}}=800 \mathrm{~A} \mathrm{~m}^{-1}$

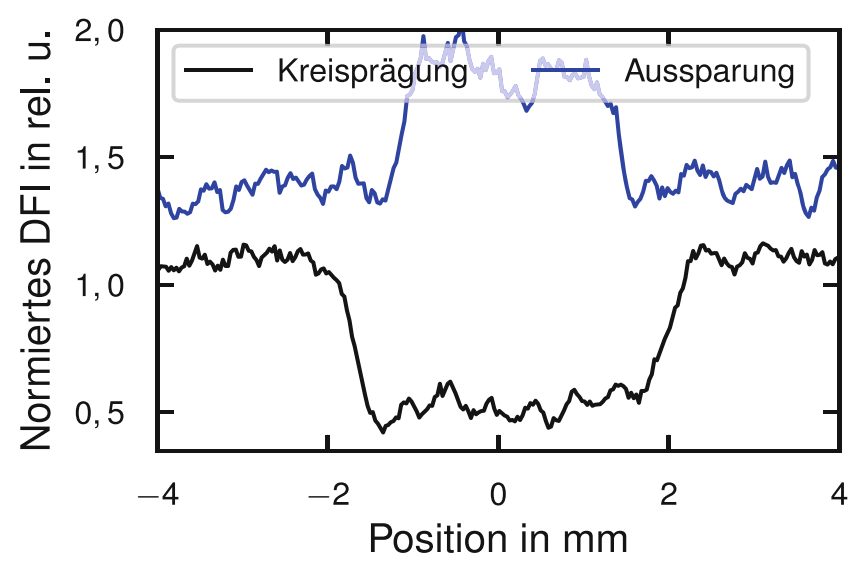

Abb. 10 Verlauf des normiertes DFI Signal in der Aussparung und Kreisprägung senkrecht zum magnetischen Feld. Der betrachtete Bereich ist in Abb. 9 mit einer roten Linie markiert

res Verhalten, da bei der ausgesparten Probe innerhalb der Barriere keine Eisenverluste auftreten können. Die Flussbarrieren der geprägten Proben bestehen aus umgeformten Werkstoff mit erhöhten Eigenspannungen, in dessen deformierten Bereichen konsequenterweise höhere Eisenverlustdichten entstehen, wie bei den homogen geprägten Proben in Abb. 5 gezeigt. Die höchsten Verluste ergeben sich interessanterweise für die mit $50 \mathrm{~N}$ am geringsten deformierten Probe. Bei den identisch mit $100 \mathrm{~N}$ Prägekraft hergestellten Proben ergibt sich ein schwankendes Bild. Eine klare Abgrenzung zur mit $200 \mathrm{~N}$ geprägten Probe ist ebenfalls nicht erkennbar. Die maximale Differenz zwischen den niedrigsten Eisenverlusten der Referenzprobe zu den Eisenverlusten der Probe Cyl. $50 \mathrm{~N}$ liegt bei $0,44 \mathrm{~W} / \mathrm{kg}$. Die beobachtete Erhöhung der Eisenverluste um maximal 10\% ist somit im Verhältnis wesentlich geringer als die gewünschte Absenkung der Permeabilität.

\subsubsection{Lokale magnetische Eigenschaften}

Mit Hilfe der nGI lässt sich die durch Eigenspannungen hervorgerufene Änderung der Trägheit magnetischer Domänen sichtbar machen. Eine veränderte Trägheit der magnetischen Domänen bedeutet gleichzeitig eine Änderung der magnetischen Permeabilität und dadurch eine Beeinflussung des magnetischen Flusses. Durch Normierung des DFI einer bearbeiteten Probe auf das DFI einer unbearbeiteten Referenz lässt sich die durch die Bearbeitung hervorgerufene Änderung des magnetischen Flusses sichtbar machen, wobei ein Signal größer (kleiner) als Eins einen erhöhten (verringerten) magnetischen Fluss in der bearbeiteten Probe im Vergleich zur Referenz bedeutet. Bei der Betrachtung der Ergebnisse muss jedoch berücksichtigt werden, dass die Kopplung zwischen Signal und Fluss komplex ist und keiner einfachen Proportionalität folgt. 
In Abb. 9 wird das normierte DFI-Signal einer Probe mit Aussparung mit dem Signal einer Probe mit Kreisprägung, wie in Abb. 3 dargestellt, verglichen. Die Prägungen wurden sequentiell mit einer Kraft von $100 \mathrm{~N}$ durchgeführt. Als Magnetfeld wurde $H_{\mathrm{m}}=800 \mathrm{~A} \mathrm{~m}^{-1}$ angelegt. Bei diesem Magnetfeld entspricht ein normiertes Signal von Eins, etwa einer Polarisation von 1,5 T (vgl. Abb. 6 a). Innerhalb der Aussparung(1) enthält das Signal keine Aussage über den magnetischen Fluss. Allerdings kann angenommen werden, dass in diesem Bereich ein vernachlässigbarer magnetischer Fluss existiert. Im Bereich(2) ist der magnetische Fluss durch die eingebrachten Eigenspannungen stark reduziert, wodurch das Signal auf 0,48 sinkt. Durch die Flussverdrängung in den Bereichen(1) und(2) erhöht sich der magnetische Fluss in den Bereichen(3) und(4). Dabei steigt im Bereich (3) das Signal auf 1,37 und im Bereich (4) auf 1,12. Die eingebrachten Eigenspannungen in Bereich (2) führen somit, ähnlich wie die Aussparung in Bereich (1), den magnetischen Fluss. Es zeigt sich, dass die Änderungen des magnetischen Flusses auf die eingebrachten Eigenspannungen und nicht auf die verringerte Blechdicke zurückzuführen sind, da auch außerhalb des geprägten Punkte ein Einfluss auf das DFI Signal sichtbar ist. Da das DFI Signal auch von der Blechdicke abhängig ist, führt eine Verringerung der Blechdicke zu einer Erhöhung des DFI Signals. Der Extremfall von Blechdicke Null lässt sich in Bereich (1) beobachten. Das heißt, eine nur von der Blechdicke abhängige Verringerung des magnetischen Flusses würde daher tendenziell zu einer Erhöhung des DFI Signals im Prägepunkt führen. Dies hat sich jedoch in keinem der nGI Bilder feststellen lassen.

In Abb. 10 wird der Verlauf des normierten DFI Signals in und um der Aussparung (blau) respektive der Kreisprägung (schwarz) senkrecht zum angelegten magnetischen Feld gezeigt. Im Graph der Kreisprägung lässt sich die Absenkung des DFI Signals in den sieben Prägelinien und das leichte Ansteigen zwischen den Prägelinien erkennen.

\section{Zusammenfassung und Ausblick}

In der vorliegenden Arbeit werden zwei verschiedene Versuchsreihen zur Quantifizierung und Verifizierung eigenspannungsbasierter magnetischer Flussbarrieren präsentiert. Hierbei wird die lokale Veränderung magnetischer Werkstoffeigenschaften von Elektroblech durch umformtechnisch induzierte Eigenspannungen untersucht. Dieses Phänomen ist die Grundlage zu einer alternativen magnetischen Flussführung in Elektromotoren bei gleichzeitig geringer Beeinflussung der mechanischen Festigkeit der Rotorkonstruktion.

Die in Abb. 1 gezeigten homogen geprägten Proben wurden genutzt, um die absolute Abnahme der magnetischen
Leitfähigkeit in geprägten Bereichen bestimmen zu können. Da der gesamte Querschnitt, der bei den SST-Messungen von magnetischem Fluss durchdrungen wird, mit Prägungen beaufschlagt ist, kann keine Verdrängung des magnetischen Flusses erfolgen. Die so gemessene magnetische Permeabilität $\mu_{\mathrm{r}}$ ist ein gutes Maß für die vorliegende Magnetisierbarkeit geprägter Blechbereiche im magnetischen Querschnitt von rotierenden elektrischen Maschinen. Die Messergebnisse zeigen eine starke Verminderung der relativen Permeabilität $\mu_{\mathrm{r}}$ auf bis zu unter $20 \%$ des Wertes der unbearbeiteten Referenzprobe. Der magnetische Widerstand steigt mit wachsender Prägekraft, die Anordnung der einzelnen Prägepunkte scheint jedoch zumindest bei der vorliegenden Auswahl der Herstellungsparameter keinen Einfluss auf die magnetischen Eigenschaften des Materials auszuüben. Eine Verdopplung der Prägepunktanzahl bei ansonsten gleichbleibenden Parametern führt ebenfalls zu einer signifikanten Erhöhung des magnetischen Widerstands. Die resultierenden Eisenverluste $P_{\mathrm{Fe}}$ wachsen mit steigender Prägekraft. Die Zunahme der Eisenverluste ist jedoch wesentlich geringer als die gewünschte Abnahme der relativen Permeabilität. In Zukunft sind weitere Untersuchungen zum Einfluss des Prägemusters unter Variation der Herstellungsparameter geplant.

Die kreisförmig geprägten Proben gemäß Abb. 3 bilden beispielhaft den späteren Anwendungsfall mit der Kombination aus geprägten und unbearbeiteten Blechbereichen ab. Die $10 \times 60 \mathrm{~mm}$ großen Proben werden ebenfalls in Bezug zu einer unbearbeiteten Referenzprobe, als auch einer Probe mit ausgeschnittenem Anteil gesetzt. Die Probe mit der kreisförmigen Aussparung stellt hierbei den herkömmlichen Ansatz von magnetischen Flussbarrieren dar. Erneut konnte eine verstärkte Reduzierung der magnetischen Leitfähigkeit gezeigt werden, die sich mit zunehmender Prägekraft immer stärker auswirkt. Wie bei den homogen geprägten Proben zeigen sich auch hier leicht erhöhte Eisenverlustdichten. Diese fallen gegenüber der Verminderung der Permeabilität jedoch nicht ins Gewicht.

Die Reproduzierbarkeit der magnetischen Eigenschaftsveränderungen durch prägungsinduzierte Eigenspannungen wurde anhand fünf identisch hergestellter Proben bei $100 \mathrm{~N}$ Prägekraft untersucht. Die geringen Schwankungen der gemessenen relativen Permeabilität liegen im akzeptablen Rahmen und betragen nicht mehr als $4 \%$ im anwendungsrelevanten Bereich zwischen 0,5 und 1,5T. Der Prozess und seine Auswirkungen auf die magnetische Materialeigenschaften kann also als stabil bewertet werden. Die durchgeführten nGI-Messungen zeigen, dass die eingebrachten Eigenspannungen nicht die gesamte Probe beeinflussen, sondern nur in der Nähe der Prägepunkte wirken, wodurch die Permeabilität lokal abgesenkt wird. Die gemessene lokale Permeabilitätsänderung ist wie gewünscht stark inhomogen und erlaubt es Prägungen ähnlich wie 
Aussparungen als Flussbarrieren zu nutzen. In zukünftigen Messkampagnen wird die Reproduzierbarkeit der Flussführung durch Prägungen neben den SST-Messungen zusätzlich mit nGI-Messungen verfiziert.

Die vorgestellte Methode zur lokalen Beeinflussung magnetischer Materialeigenschaften bietet großes Potential zur Optimierung und Anpassung von Rotor-Topologien elektrischer Antriebe. Weitere Untersuchungen werden die Auslegung geeigneter Prägegeometrien und -muster sowie die numerische Auslegung geprägter magnetischer Flussbarrieren thematisieren, um die gewonnen Erkenntnisse in den Auslegungsprozess rotierender elektrischer Maschinen einsetzen zu können.

Funding Open Access funding enabled and organized by Projekt DEAL.

Open Access Dieser Artikel wird unter der Creative Commons Namensnennung 4.0 International Lizenz veröffentlicht, welche die Nutzung, Vervielfältigung, Bearbeitung, Verbreitung und Wiedergabe in jeglichem Medium und Format erlaubt, sofern Sie den/die ursprünglichen Autor(en) und die Quelle ordnungsgemäß nennen, einen Link zur Creative Commons Lizenz beifügen und angeben, ob Änderungen vorgenommen wurden.

Die in diesem Artikel enthaltenen Bilder und sonstiges Drittmaterial unterliegen ebenfalls der genannten Creative Commons Lizenz, sofern sich aus der Abbildungslegende nichts anderes ergibt. Sofern das betreffende Material nicht unter der genannten Creative Commons Lizenz steht und die betreffende Handlung nicht nach gesetzlichen Vorschriften erlaubt ist, ist für die oben aufgeführten Weiterverwendungen des Materials die Einwilligung des jeweiligen Rechteinhabers einzuholen.

Weitere Details zur Lizenz entnehmen Sie bitte der Lizenzinformation auf http://creativecommons.org/licenses/by/4.0/deed.de.

\section{Literatur}

1. Reddy PB, El-Refaie AM, Galioto S, Alexander JP (2017) Design of synchronous reluctance motor utilizing dual-phase material for traction applications. IEEE Trans Ind Appl 53:1948-1957
2. Chai F, Li Y, Liang P, Pei Y (2016) Calculation of the maximum mechanical stress on the rotor of interior permanent-magnet synchronous motors. IEEE Trans Ind Electron 63:3420-3432

3. Babetto C, Bacco G, Bianchi N (2018) Synchronous reluctance machine optimization for high-speed applications. IEEE Trans Energy Convers 33(3): 1266-1273

4. Weiss HA, Leuning N, Steentjes S, Hameyer K, Andorfer T, Jenner S, Volk W (2017) Influence of shear cutting parameters on the electromagnetic properties of non-oriented electrical steel sheets. J Magn Magn Mater 421:250-259

5. Hofmann M, Naumoski H, Herr U, Herzog HG (2015) Magnetic properties of electrical steel sheets in respect of cutting: micromagnetic analysis and macromagnetic modeling. IEEE Trans Magn 52(2): $1-14$

6. Moll I, Vogt S, Neuwirth T, Schauerte B, Hameyer K, Schulz M, Gustschin A, Volk W, Weiss HA (2020) Analysis of cylindrically and spherically embossed flux barriers in non-oriented electrical steel. In: The 13th International Conference on the Technology of Plasticity

7. Vogt S, Neuwirth T, Schauerte B, Weiss HA, Falger PM, Gustschin A, Schulz M, Hameyer K, Volk W (2019) Extent of embossing-related residual stress on the magnetic properties evaluated using neutron grating interferometry and single sheet test. Prod Eng 13:211-217

8. Fiorillo F (2010) Measurements of magnetic materials. Metrologia 47:114

9. Grünzweig C, David C, Bunk O, Dierolf M, Frei G, Kühne G, Kohlbrecher J, Schäfer R, Lejcek P, Rønnow HMR et al (2008) Neutron decoherence imaging for visualizing bulk magnetic domain structures. Phys Rev Lett 101(2):25504

10. Neuwirth T, Backs A, Gustschin A, Vogt S, Pfeiffer F, Böni P, Schulz M (2020) A high visibility Talbot-Lau neutron grating interferometer to investigate stress-induced magnetic degradation in electrical steel. Sci Rep 10(1):1-12

11. Calzada E, Gruenauer F, Mühlbauer M, Schillinger B, Schulz M (2009) New design for the ANTARES-II facility for neutron imaging at FRM II. Nucl Instrum Methods Phys Res A 605(1/2):50-53

12. Schulz M, Schillinger B (2015) ANTARES: cold neutron radiography and tomography facility. JLSRF $1: 17$ 